\title{
LIMIAR ANAERÓBICO VENTILATÓRIO EM ADOLESCENTES BRASILEIROS DE AMBOS OS SEXOS'
}

\author{
MS. KAMILLA BOLONHA GOMES \\ Mestre em Educação Física pelo Centro de Educação Física e Desportos da Universidade Federal do \\ Espírito Santo (CEFD/UFES), Professora de Educação Física do Programa de Orientação ao Exercício \\ Físico (PROEF - PMS/ES) (Serra - Espírito Santo - Brasil) \\ E-mail: kamillabolonha@yahoo.com.br
}

\section{DRA. LUCIANA CARLETTI}

Doutora em Ciências Fisiológicas, Professora do Programa de Pós-Graduação em Educação Física da Universidade Federal do Espírito Santo (UFES), Laboratório de Fisiologia do Exercício - LAFEX/CEFD/ UFES (Vitória - Espírito Santo - Brasil)

E-mail: Icarletti@terra.com.br

\section{DR. ANSELMO JOSÉ PEREZ}

Doutor em Ciências Fisiológicas, Professor do Programa de Pós-Graduação em Educação Física da Universidade Federal do Espírito Santo (UFES), Laboratório de Fisiologia do Exercício

- LAFEX/CEFD/UFES (Vitória - Espírito Santo - Brasil)

E-mail: anselmo@cefd.ufes.br

\section{DR. ANABEL NUNES RODRIGUES}

Doutor em Ciências Fisiológicas, Professor do Centro Universitário do Espírito Santo (UNESC)

(Colatina - Espírito Santo - Brasil)

E-mail: anrodrigues@unesc.br

\section{RESUMO}

Com o objetivo de comparar as respostas cardiorrespiratórias no limiar anaeróbico ventilatório (LAV) em relação ao gênero, faixa etária e dados publicados na literatura, foram avaliados 289 adolescentes, de ambos os sexos, entre 10 e 14 anos. Foi realizado um teste cardiopulmonar, utilizando o protocolo de rampa em esteira ergométrica. O LAV foi identificado pelos métodos do V-slope e/ou do equivalente ventilatório de oxigênio. Os resultados mostraram que o consumo de oxigênio $\left(\mathrm{VO}_{2 \text { LAV }}\right.$ ) absoluto foi maior nos meninos aos 13 e 14 anos; o VO 2 LAV relativo ao peso corporal foi diferente entre os gêneros apenas aos 14 anos, sendo maior nos meninos. Conclui-se que há perda da aptidão física aeróbica dos adolescentes, de ambos os sexos, a partir dos 12 anos, com valores inferiores comparados à literatura.

PALAVRAS-CHAVE: Consumo de oxigênio; adolescente; aptidão física; limiar anaeróbio.

1. Este estudo foi financiado pelo Fundo de Apoio à Ciência e Tecnologia (FACITEC) - Prefeitura Municipal de Vitória/ ES, e pela Fundação de Amparo à Pesquisa do Espírito Santo (FAPES) (modalidade bolsa de mestrado). 
A aptidão aeróbica ou cardiorrespiratória tem sido utilizada como índice para aptidão física e desempenho esportivo, e pode ser definida como a capacidade máxima que pulmões, coração e sangue têm para fornecer e transportar oxigênio, e os tecidos e órgãos de extrair e utilizá-lo para produção de energia durante o esforço físico, o que depende da interação das respostas fisiológicas dos sistemas cardiovascular e respiratório (WASSERMAN et al., 2005; O'DONOVAN et al., 20 I 0).

Para análise e interpretação das variáveis do sistema cardiorrespiratório tem-se utilizado testes de esforço físico com carga progressiva e mensuração da troca gasosa, seja em esteira ou em cicloergômetro. Myers e Bellin (2000) mostraram que o teste de rampa, com aumento contínuo da carga de trabalho, é vantajoso quando comparado a testes tradicionais, pois apresenta boa previsão do consumo de oxigênio, em avaliações de respostas máximas ou submáximas do exercício. A vantagem para o teste de rampa também foi apresentada para crianças e adolescentes brasileiros (SILVA; SARAIVA; SOBRAL, 2007).

Assim, a aptidão cardiorrespiratória pode ser avaliada tanto pelo consumo máximo de oxigênio $\left(\mathrm{VO}_{2}\right.$ máx.), apresentado de forma absoluta ou relativa, como pelo limiar anaeróbico ventilatório (LAV), que é conceituado por Wasserman et al. (2005) como o nível de $\mathrm{VO}_{2}$ do exercício acima do qual a produção de energia aeróbica é suplementada pelos mecanismos anaeróbicos que é refletido por um aumento na relação lactato e lactato/piruvato no músculo e sangue arterial.

$\mathrm{O} \mathrm{VO}_{2}$ máx. tem sido uma das capacidades do sistema cardiorrespiratório mais estudadas nos últimos anos, e amplamente reconhecido como o melhor índice para avaliar a aptidão aeróbica, tanto em adultos (ASTRAND; RODAHL, 1986), como em crianças e adolescentes (ARMSTRONG; WELSMAN, 200 I; CHINTALA; EPSTEIN; SINGH, 2008; GUERRERO; NARANJO; CARRANZA, 2008; ARMSTRONG; TOMKINSON; EKELUND, 20l I).

O LAV, por sua vez, é considerado um marcador confiável de aptidão aeróbica submáxima devido à sua capacidade de ser sustentado por um longo período de tempo e de sua sensibilidade ao descondicionamento aeróbico e sedentarismo (WASSERMAN et al., 1999) e, por esse motivo, também tem sido bastante estudado (REYBROUCK et al., I 985; ZANCONATO et al., I 989; PEREZ; CARLETTI, 2012). Bar-Or ( 1983, p. 15) sugere ainda que entre pré-púberes, o LAV pode refletir melhor condicionamento relacionado às melhorias na potência aeróbica máxima do que o $\mathrm{VO}_{2}$ máx.

Valores normais de respostas cardiorrespiratórias ao exercício máximo para crianças e adolescentes já foram amplamente publicados (CUNNINGHAM et al., 
1984; REYBROUCK et al., 1985; ZANCONATO et al., 1989; WASHINGTON et al., 1988; ROWLAND; CUNNINGHAM, 1992, 1997; AL-HAZZAA, 200 I; MANDADZHIEVA et al., 2005; RODRIGUES et al., 2006; SILVA; SARAIVA; SOBRAL, 2007). Contudo, alguns desses estudos apontam também valores para - LAV, que podem ser mencionados como referências, baseados em amostras internacionais, para avaliar o nível de resistência cardiorrespiratória de crianças e adolescentes no exercício submáximo (REYBROUCK et al., I 985; ZANCONATO et al., 1989; AL-HAZZAA, 200 I).

Sabe-se que no período da adolescência ocorrem aumentos no crescimento longitudinal e na estrutura dos órgãos, bem como adaptações no desenvolvimento dos sistemas fisiológicos, que irão refletir no consumo de oxigênio do esforço máximo e submáximo (ROWLAND, 2008).

Assim, a importância de se conhecer a aptidão cardiorrespiratória de adolescentes está relacionada tanto à saúde, em que uma maior aptidão pode estar correlacionada a um menor perfil de risco cardiovascular (RODRIGUES et al., 2006, 2007), como à prescrição de exercícios e ao efeito do treinamento (ROWLAND; DELANEY; SICONOLFI, 1987; ARMSTRONG; WELSMAN, 1994; PRADO et al., 2009, 2010).

Embora estudos internacionais já tenham apresentado valores de referência para o LAV em adolescentes, até o momento a literatura nacional é limitada em relação às amostras, considerando o tamanho e a característica das mesmas (BRUNETTO et al., 2005; STELLA et al., 2005).

Dessa forma, a proposta desse estudo é apresentar as respostas cardiorrespiratórias no LAV de adolescentes entre 10 e 14 anos, analisando e comparando-os em relação ao gênero, à faixa etária e a dados publicados na literatura, contribuindo para a criação de valores de referência para essa população.

\section{MATERIAL E MÉTODOS}

\section{AMOSTRA}

A amostra foi constituída de adolescentes saudáveis de ambos os sexos na faixa etária entre 10 e 14 anos (WHO, 1995), estudantes da rede municipal de ensino de Vitória/ES.

Para o cálculo amostral utilizou-se a equação geral para grandes e pequenas populações (REA; PARKER, 2000), com um nível de confiança de 95\% e intervalo de confiança de $5 \%$.

A amostra foi obtida por um processo de seleção amostral aleatória nas sete regiões administrativas do município de Vitória/ES. As escolas foram sorteadas 
e convidadas a participarem por meio de seus diretores, que forneceram uma listagem de seus alunos. A partir dessa lista foram sorteados 300 estudantes. Os adolescentes sorteados foram convidados, por meio de seus pais ou responsáveis, a participarem do estudo. Todos os participantes assinaram o termo de consentimento livre e esclarecido. $O$ protocolo de estudo foi aprovado pelo comitê de ética em pesquisa da Faculdade Salesiana de Vitória, por meio do ofício n 069/2007, com registro no CEP sob o n 053/2007.

Foram considerados critérios de exclusão: ser tabagista e portador de alguma doença metabólica previamente conhecida.

\section{MEDIDAS ANTROPOMÉTRICAS E MATURAÇÃO SEXUAL}

A massa corporal e a estatura foram medidas utilizando uma balança eletrônica antropométrica (Welmy, modelo RW200; São Paulo, BR) com precisão de I 00 gramas (g) e capacidade máxima para I 50 quilos (kg), e um estadiômetro de madeira (Seca, modelo 206) com escala de 0, I centímetros (cm), respectivamente. A partir dessas duas variáveis, calculou-se o índice de massa corporal (IMC, kg/m²).

Como intuito de apresentar características de maturação sexual da amostra, foram utilizados os critérios propostos por Duarte ( 1993). Assim, as meninas foram consideradas maturadas quando já havia ocorrido a menarca, e os meninos, pela presença de pelos axilares.

\section{PROTOCOLO DO TESTE DE ESFORÇO CARDIOPULMONAR}

Os adolescentes realizaram um teste de esforço cardiopulmonar em esteira ergométrica (Inbrasport Super ATL, Rio Grande do Sul, BR), utilizando o analisador de gases da marca MedGraphics Corporation (MGC, modelo CPX/D; Minnesota, EUA), o qual fornecia informações acerca do consumo de oxigênio $\left(\mathrm{VO}_{2}\right)$, produção de dióxido de carbono $\left(\mathrm{VCO}_{2}\right)$, ventilação pulmonar $(\mathrm{VE})$, equivalente ventilatório de oxigênio $\left(\mathrm{VENO}_{2}\right)$ e equivalente ventilatório de dióxido de carbônico $\left(\mathrm{VENCO} \mathrm{N}_{2}\right)$ e razão de troca respiratória $\left(\mathrm{RTR}=\mathrm{VCO}_{2} \mathrm{NO}_{2}\right)$.

Inicialmente, todos os voluntários foram submetidos a um eletrocardiograma de repouso, utilizando as 12 derivações convencionais. Os adolescentes permaneciam em decúbito dorsal por cerca de 5 minutos, em um ambiente tranquilo, com temperatura mantida em torno de $22^{\circ} \mathrm{C}$, com posterior registro da atividade elétrica do coração. Em seguida, os adolescentes eram encaminhados para a esteira ergométrica e orientados a respeito do desenvolvimento do teste e critérios para a interrupção. 
Uma máscara de neoprene, conectada ao pneumotacômetro para medida do fluxo de ar e análise dos gases expirados, foi utilizada de acordo com o tamanho do rosto de cada adolescente avaliado, permitindo a respiração pela boca e pelo nariz.

O teste era iniciado após 2 minutos de repouso em pé realizando os registros eletrocardiográficos e ventilatórios. Durante a execução, os indivíduos eram monitorados por meio de eletrocardiograma (ECG) de 12 derivações (Elite/ Micromed, Brasília, Brasil), para acompanhar a resposta eletrocardiográfica de esforço. Foi utilizado o protocolo de rampa, com aumento progressivo da carga de trabalho (velocidade - km/h, e inclinação da esteira - percentual), evoluindo de acordo com $\circ \mathrm{VO}_{2}$ máx. predito em equivalentes metabólicos (MET), comparado com o $\mathrm{VO}_{2}$ máx. medido.

Quando o indivíduo atingia a velocidade máxima predita sem que estivesse em fadiga, a inclinação era aumentada com incremento gradual de 0,5\% a cada 45 segundos. $\bigcirc$ teste era interrompido se o avaliado sinalizasse (através de gestos pré-combinados) para a fadiga, ou algum desconforto que o impedisse na continuidade do mesmo. Foram considerados máximos os testes que atenderam aos seguintes critérios (ROWLAND; CUNNINGHAM, 1992): a) frequência cardíaca máxima atingida > 190 bpm; e b) RTR $>1,0$.

\section{COLETA E ANÁLISE DOS GASES}

analisador de gases citado anteriormente consiste em um sistema de calorimetria de circuito aberto, ou seja, o gás de referência para calibração era ajustado por uma mistura de gases com concentração constante de $\mathrm{CO}_{2}$ e $\mathrm{O}_{2}$. O software Breeze Suite (MGC) era utilizado para determinação da concentração do $\mathrm{VO}_{2}$ e $\mathrm{VCO}_{2}$ pela medida da VE (volume minuto ou volume expirado de ventilação por minuto) e da diferença entre as pressões gasosas no ar inspirado e no ar expirado aferidos a cada respiração (breath by breath). Um transdutor fazia quantificação da concentração do $\mathrm{CO}_{2}$ através de análise infravermelha não dispersiva (NDIR), e outro fazia a quantificação do $\mathrm{O}_{2}$ por meio de célula de zircônio. A RTR foi calculada a partir da relação entre $\mathrm{VCO}_{2}$ e $\mathrm{VO}_{2}$ a cada incursão respiratória.

O equipamento era calibrado antes do teste da seguinte forma: aguardava-se 30 minutos para o aquecimento do circuito; em seguida, ajustava-se o fluxo de ar através do uso de uma seringa de calibração com capacidade de gerar um volume de 3 litros (L); a seringa era manipulada manualmente para produzir um fluxo de 0,4 a $12 \mathrm{~L} / \mathrm{s}$, para verificar a calibração do sistema para fluxo baixo e alto; o sensor de $\mathrm{O}_{2}$ e $\mathrm{CO}_{2}$ era calibrado usando como referência um gás composto de $22 \%$ de $\mathrm{O}_{2}$ e $0 \%$ de $\mathrm{CO}_{2}$; posteriormente, era calibrado com o uso de um gás composto de $12 \%$ de $\mathrm{O}_{2}$ e $5 \%$ de $\mathrm{CO}_{2}$. 
Para cada indivíduo foram construídos três gráficos: V-slope, cuja técnica consiste na determinação do ponto de quebra da linearidade da inclinação da $\mathrm{VCO}_{2}$ versus $\mathrm{VO}_{2}$; $\mathrm{VENO}_{2}$ e VENCO ; e RTR.

A identificação do LAV foi realizada pelo método visual, de acordo com os seguintes critérios (BEAVER; WASSERMAN; WHIPP, 1986): ponto de quebra da linearidade da inclinação de $\mathrm{VCO}_{2}$ versus $\mathrm{VO}_{2}$ (método $\mathrm{V}$-slope); e/ou aumento na relação $\mathrm{VENO} \mathrm{N}_{2}$ sem aumento concomitante na relação $\mathrm{VENCO}$.

Os gráficos, construídos utilizando o programa Microsoft Office Excel 2007, foram analisados por dois avaliadores experientes para confirmar a identificação da variável. Havendo discordância, um terceiro avaliador era requisitado.

\section{VARIÁVEIS ANALISADAS DO TESTE CARDIOPULMONAR}

As seguintes variáveis dependentes foram medidas respiração a respiração: $\mathrm{VO}_{2}\left(\mathrm{~L} \cdot \mathrm{min}^{-1}\right.$ e $\left.\mathrm{ml} \cdot \mathrm{kg}^{-1} \cdot \mathrm{min}^{-1}\right) ; \mathrm{VCO}_{2}\left(\mathrm{~L} \cdot \mathrm{min}^{-1}\right) ; \mathrm{RTR} ; \mathrm{VE}\left(\mathrm{L} \cdot \mathrm{min}^{-1} \mathrm{BTPS}\right)$; frequência cardíaca (FC bpm); velocidade (Vel LAV km/h) e inclinação (Incl LAV percentual - \%). Foram calculados os percentuais do $\mathrm{VO}_{2}$ máx. $\left(\% \mathrm{VO}_{2}\right.$ máx.) e da $\mathrm{FC}$ máxima (\%FCmáx.).

\section{ANÁLISE ESTATIISTICA}

Para a análise estatística (SigmaStat 3.5) foram utilizados recursos da estatística descritiva (média, desvio padrão) para as medidas antropométricas e para as variáveis cardiorrespiratórias no LAV nas faixas etárias e gêneros.

Utilizou-se a análise de variância (ANOVA) de uma via para as comparações das variáveis cardiorrespiratórias e da variável relacionada à carga de trabalho entre as faixas etárias e para cada gênero. $\bigcirc$ teste de post hoc de Tukey foi usado para comparar as diferenças entre os grupos, e o teste de Dunn, quando os dados não passaram pela normalidade. Para as comparações entre os gêneros foi aplicado o teste $t$ de Student para amostras independentes. $\bigcirc$ nível de $p<0,05$ foi usado para determinar as significâncias estatísticas.

\section{RESULTADOS}

No total, 289 adolescentes atenderam aos critérios de inclusão para teste máximo e identificação do LAV, os quais foram divididos de acordo com a faixa etária e gênero. 
As características antropométricas dos grupos etários são apresentadas na Tabela I. Todos os adolescentes foram classificados como peso normal a partir dos valores de referência propostos pela Organização Mundial da Saúde (WHO, 2007), que utiliza os valores médios de IMC para idade e sexo.

Observam-se valores significativamente maiores da massa corporal para as meninas a partir dos 12 anos e da estatura a partir dos II anos. Já para os meninos esse fato ocorreu a partir dos 13 e 12 anos, respectivamente.

Tabela I - Características antropométricas de meninas e meninos nas divisões etárias

\begin{tabular}{|c|c|c|c|c|c|c|c|c|c|}
\hline \multirow{2}{*}{$\begin{array}{l}\text { Idade } \\
\text { (anos) }\end{array}$} & \multirow{2}{*}{$n$} & \multicolumn{4}{|c|}{ Meninas } & \multicolumn{4}{|c|}{ Meninos } \\
\hline & & $\mathrm{n}$ & $M C(k g)$ & Estatura (m) & $\mathrm{IMC}\left(\mathrm{kg} / \mathrm{m}^{2}\right)$ & $\mathrm{n}$ & $M C(k g)$ & Estatura (m) & $\mathrm{IMC}\left(\mathrm{kg} / \mathrm{m}^{2}\right)$ \\
\hline 10 & 43 & 26 & $34,5 \pm 6,8$ & $|, 4| \pm 0,06$ & $17,3 \pm 2,6$ & 17 & $33,3 \pm 7,7$ & $1,40 \pm 0,07$ & $16,9 \pm 2,8$ \\
\hline 11 & 70 & 44 & $42,1 \pm 11,8$ & $1,47 \pm 0,08$ & $19,2 \pm 3,8 *$ & 26 & $36,8 \pm 8,3$ & $1,46 \pm 0,07$ & $17,2 \pm 2,8$ \\
\hline 12 & 54 & 29 & $43,0 \pm 8,5 * \mathrm{e}$ & $1,52 \pm 0,07^{* g}$ & $18,5 \pm 2,8$ & 25 & $38,1 \pm 8,7$ & $1,48 \pm 0,06^{e}$ & $17,3 \pm 3,0$ \\
\hline 13 & 59 & 33 & $46,0 \pm 8,8^{d}$ & $1,55 \pm 0,08^{f}$ & $18,9 \pm 2,7$ & 26 & $52,8 \pm 11,6^{* b}$ & $1,63 \pm 0,08^{* b}$ & $19,9 \pm 3,6^{b}$ \\
\hline 14 & 63 & 26 & $52,6 \pm 12,9$ & $1,60 \pm 0,06^{a}$ & $20,6 \pm 4,6 * h$ & 37 & $50,0 \pm 11,6^{a}$ & $1,62 \pm 0,09^{a}$ & $|8,8 \pm 3|$, \\
\hline
\end{tabular}

IMC - índice de massa corporal; MC - massa corporal; média \pm desvio padrão; * $p<0,05$ entre gêneros; $p<0,05$ faixas etárias: ${ }^{\mathrm{a}} \mid 4>10,11$ e $12 ;{ }^{\mathrm{b}} 13>10,11$ e $12 ;^{\mathrm{c}} 14>10$ e $11 ;^{\mathrm{d}} 13>10 ;{ }^{\mathrm{e}} 12>10 ;^{\mathrm{f}} 13>10$ e $11 ;{ }^{\mathrm{g}} 12$ $>10$ e $11 ;^{h} 14>10 ;{ }^{i} 11>10$.

Ao comparar os gêneros, as meninas de 12 anos apresentaram valores maiores para massa corporal e estatura quando comparadas com os meninos. Os meninos mostraram maior massa corporal e estatura aos 13 anos. Nas demais faixas etárias, não foram encontradas diferenças significativas.

Com relação à característica maturacional observada pela menarca nas meninas, nenhuma havia atingido aos 10 anos, 7 (4\%) aos II anos, 13 (8\%) aos 12 anos, 24 (15\%) aos 13 anos e 25 (16\%) aos 14 anos. Para os meninos a presença de pelos axilares foi notada em ocorrências de: I (I\%) aos I0 anos, 3 (2\%) aos I I anos, 4 (3\%) aos 12 anos, 21 (16\%) aos 13 anos e 27 (21\%) aos 14 anos.

Na Tabela 2 estão as variáveis no LAV, de ambos os gêneros, de acordo com as faixas etárias. São elas: $\mathrm{VO}_{2 \text { LAV }}\left(\mathrm{L} \cdot \mathrm{min}^{-1}\right)$ e $\left(\mathrm{ml} \cdot \mathrm{kg}^{-1} \cdot \mathrm{min}^{-1}\right), \% \mathrm{VO}_{2}$ máx., FC LAV e \%FCmáx. 
Tabela 2. Valores de consumo de oxigênio $\left(\mathrm{VO}_{2}\right)$ e frequência cardíaca (FC) no LAV para ambos os sexos

\begin{tabular}{|c|c|c|c|c|c|c|}
\hline $\begin{array}{l}\text { Idade } \\
\text { (anos) }\end{array}$ & $\begin{array}{c}\text { Gêne- } \\
\text { ro }\end{array}$ & $\mathrm{VO}_{2 \operatorname{LAV}}\left(\mathrm{L} \cdot \mathrm{min}^{-1}\right)$ & $\mathrm{VO}_{2 \text { LAV }}\left(\mathrm{ml} \mathrm{kg}^{-1} \cdot \mathrm{min}^{-1}\right)$ & \% VO ${ }_{2}$ máx. & $\mathrm{FC}_{\mathrm{LAV}}(\mathrm{bpm})$ & \%FCmáx. \\
\hline \multirow{2}{*}{10} & $\mathrm{~F}$ & $0,65 \pm 0,23$ & $19,3 \pm 7,0$ & $50,6 \pm 17,4$ & $|48 \pm 2|^{j}$ & $74,0 \pm 11$ \\
\hline & M & $0,7 \mid \pm 0,30$ & $21,0 \pm 6,4$ & $48,0 \pm 16,0$ & $|37 \pm 2|$ & $70,0 \pm 12$ \\
\hline \multirow{2}{*}{11} & $\mathrm{~F}$ & $0,72 \pm 0,19$ & $17,6 \pm 5,4$ & $45,6 \pm 12,8$ & $135 \pm 17$ & $68,2 \pm 8,8$ \\
\hline & M & $0,70 \pm 0,22$ & $19,8 \pm 7,5$ & $43,8 \pm 13,3$ & $138 \pm 18$ & $69,5 \pm 8,9$ \\
\hline \multirow{2}{*}{12} & $\mathrm{~F}$ & $0,78 \pm 0,24$ & $18,0 \pm 3,9$ & $47,7 \pm 11,4$ & $145 \pm 13$ & $72,3 \pm 6,9$ \\
\hline & M & $0,73 \pm 0,22$ & $19,7 \pm 6,0$ & $43,7 \pm 10,6$ & $139 \pm 19$ & $70,1 \pm 9,8$ \\
\hline \multirow{2}{*}{13} & $\mathrm{~F}$ & $0,84 \pm 0,33$ & $\mid 8,1 \pm 6,0$ & $47,1 \pm 13,1$ & $142 \pm 19$ & $71,4 \pm 8,8$ \\
\hline & M & $|, 0| \pm 0,3 \mid$ *b & $20,0 \pm 7,9$ & $43,3 \pm 11,7$ & $139 \pm 16$ & $69,5 \pm 7,8$ \\
\hline \multirow{2}{*}{14} & $\mathrm{~F}$ & $0,81 \pm 0,23$ & $15,9 \pm 5,5$ & $43,2 \pm 11,8$ & $140 \pm 18$ & $70,6 \pm 10$ \\
\hline & M & $1,21 \pm 0,49 * a$ & $24,9 \pm 9,8 *$ & $48,9 \pm 18,1$ & $140 \pm 22$ & $70,2 \pm 11$ \\
\hline
\end{tabular}

Média \pm desvio padrão; LAV - limiar anaeróbico ventilatório; $F$ - feminino; $M$ - masculino; * $p<0,05$ entre gêneros; $p<0,05$ faixas etárias: ${ }^{a} \mid 4>10,11$ e $12 ;{ }^{b} 13>10,11$ e $12 ;{ }^{i} 10>11$.

Na comparação entre as faixas etárias, podemos observar que no grupo das meninas não foram encontradas diferenças estatisticamente significantes para as variáveis anteriormente citadas, exceto para a $\mathrm{FC}_{\text {LAV }}$ onde aos 10 anos apresentou maior média quando comparada aos II anos.

A análise do grupo dos meninos revela diferenças estatísticas para o $\mathrm{VO}_{2 \text { LAV }}$ $\left(\mathrm{L}\right.$. $\left.\mathrm{min}^{-1}\right)$, onde os meninos de 13 e 14 anos apresentaram valores maiores quando comparados aos de 10, II e 12 anos, não havendo diferença entre os 13 e 14 anos, e nem entre as demais idades.

Ainda na Tabela 2, para a comparação entre os gêneros, foram observadas diferenças estatísticas no $\mathrm{VO}_{2 \text { LAV }}$ Em valores absolutos o $\mathrm{VO}_{2 \text { LAV }}\left(\mathrm{L}\right.$. $\left.\mathrm{min}^{-1}\right)$ foi superior para os meninos nas idades de 13 e 14 anos, e para o $\mathrm{VO}_{2 \text { LAV }}$ relativo à massa corporal $\left(\mathrm{ml} \cdot \mathrm{kg}^{-1} \cdot \mathrm{min}^{-1}\right)$ os resultados foram maiores nos meninos apenas aos 14 anos. Para $\circ \% \mathrm{VO}_{2}$ máx. não foram identificadas diferenças entre as faixas etárias e nem mesmo entre os gêneros.

$\mathrm{Na}$ análise dos resultados da velocidade $\left(\mathrm{Vel}{ }_{\text {LAV }} \mathrm{km} / \mathrm{h}\right.$ ) e da inclinação (Incl LAV \%) entre as faixas etárias, em cada gênero, observamos que os meninos de 14 anos apresentaram valores estatisticamente maiores para Vel ${ }_{\text {LAV }}(7,9 \pm 1,1 \mathrm{~km} / \mathrm{h})$ em comparação às demais idades ( 10,11 e 12 anos), exceto para os de 13 anos $(7,4 \pm 1,1$ km/h). Não houve diferença entre as demais faixas etárias. Também não foram demonstradas diferenças significativas para os valores de Ind LAV No grupo 
das meninas, não foram encontradas diferenças significativas entre as faixas etárias para as cargas de trabalho no LAV, onde a média da Vel ${ }_{\text {LAV }}$ para a faixa etária de 10 a 14 anos foi de $6,8 \pm 0,2 \mathrm{~km} / \mathrm{h}$ e Incl ${ }_{\text {LAV }}$ de $0,3 \pm 0,1 \%$.

A análise comparativa entre gêneros revelou valores estatisticamente maiores da Vel LAV para os meninos de II, 13 e 14 anos, e da Ind LAV apenas aos 14 anos.

\section{DISCUSSÃO}

A proposta desse estudo foi apresentar as respostas cardiorrespiratórias, em níveis submáximos de esforço representado pelo LAV, de adolescentes entre 10 e 14 anos, analisando-as e comparando-as em relação ao gênero, à faixa etária e à literatura.

Dessa forma, os resultados sugerem que a capacidade cardiorrespiratória obtida pelo $\mathrm{VO}_{2}$ relativo ao peso corporal no LAV foi semelhante entre meninos e meninas até os 13 anos. Após essa idade, os meninos apresentaram valores mais elevados, sugerindo uma melhora na aptidão aeróbica submáxima.

O maior LAV nos adolescentes mais velhos do sexo masculino, não acompanhado de elevação no percentual do $\mathrm{VO}_{2}$ máx., em comparação com o feminino, sugere melhorias no sistema aeróbico. Esse resultado é corroborado pela literatura, que atribui essas mudanças ao aumento das câmaras ventriculares, do tamanho pulmonar, da massa muscular, da capilarização dos músculos, da capacidade das enzimas oxidativas e da concentração de hemoglobina, resultando em maior disponibilidade no conteúdo de oxigênio plasmático, especialmente em meninos (ROWLAND et al., 2008). No entanto, ainda existem conflitos na literatura sobre os processos fisiológicos e as melhorias de aptidão aeróbica em relação ao processo de crescimento e maturação nessa faixa etária (ARMSTRONG; TOMKINSON; EKELUND, 201 I). Assim, as inferências dos nossos dados devem ser feitas com cautela.

Embora Armstrong, Welsman e Kirby (1999) tenham sugerido que a maturação biológica não influencie a resposta do $\mathrm{VO}_{2}$ no exercício submáximo, Bar-Or ( 1983 ) relatou que não somente as dimensões corporais, mas também o estágio maturacional está diretamente relacionado às mudanças fisiológicas na adolescência. Essas discrepâncias denotam as dificuldades de delineamento metodológico dos estudos que abordaram a temática da maturação biológica (RATEL et al., 20 l 0).

A observação dos estratos etários revelou que, nos meninos, o aumento da idade representou poucas diferenças nas variáveis estudadas. Quando analisado o $\mathrm{VO}_{2 \text { LAV }}\left(\mathrm{ml} \mathrm{kg}^{-1} \cdot \mathrm{min}^{-1}\right)$, os meninos mostraram uma tendência ao aumento dessa variável apenas aos 14 anos, contudo não houve diferenças estatísticas. Resultados semelhantes, no que se refere ao comportamento dessa variável, foram encontrados 
no estudo de Al-Hazzaa (200I), onde os valores para $\mathrm{VO}_{2 \text { LAV }}\left(\mathrm{ml} . \mathrm{kg}^{-1} \cdot \mathrm{min}^{-1}\right)$ refletiam o nível de aptidão de meninos, que variou entre $32 \pm 5,2 \mathrm{ml} \cdot \mathrm{kg}^{-1} \cdot \mathrm{min}^{-1}$ aos 9-II anos, 34,4 \pm 6,2 ml. $\mathrm{kg}^{-1} \cdot \mathrm{min}^{-1}$ aos II-13 anos e 38,8 \pm 7,3 $\mathrm{ml} \cdot \mathrm{kg}^{-1} \cdot \mathrm{min}^{-1}$ aos 13 - 15 anos.

Em relação ao processo de crescimento e desenvolvimento, Bar-Or ( 1983) relatou que $\mathrm{VVO}_{2}$ máx. $\left(\mathrm{L} \cdot \mathrm{min}^{-1}\right)$ aumentou concomitantemente ao longo da infância e da adolescência acompanhando o crescimento corporal, e que até os 12 anos os valores cresciam na mesma proporção em ambos os gêneros, embora os meninos tivessem valores mais elevados desde os 5 anos. Nesse sentido, Rodrigues et al. (2006) mostraram que o comportamento do $\mathrm{VO}_{2}$ máx. em adolescentes foi ascendente para os meninos (entre 43,53 $\pm 6,65 \mathrm{ml} \cdot \mathrm{kg}^{-1} \cdot \mathrm{min}^{-1}$ aos 10 anos e 49,55 $\pm 7,29 \mathrm{ml} \cdot \mathrm{kg}^{-1} \cdot \mathrm{min}^{-1}$ aos 14 anos) e descendente para as meninas (entre 38,29 $\pm 6,28 \mathrm{ml} \cdot \mathrm{kg}^{-1} \cdot \mathrm{min}^{-1}$ aos 10 anos e 36,76 $\pm 5,98 \mathrm{ml} \cdot \mathrm{kg}^{-1} \cdot \mathrm{min}^{-1}$ aos 14 anos). De acordo com Armstrong, Tomkinson e Ekelund (20 I I), há elevação do pico de $\mathrm{VO}_{2}$ com o avanço da idade dos 8 aos 16 anos em até I50\% para os meninos, e um aumento de $80 \%$ para as meninas até aproximadamente os 13 anos, e estabilização ou queda após essa idade.

Dessa forma, esperava-se neste estudo que esse comportamento fosse o mesmo para os resultados do $\mathrm{VO}_{2 \text { LAV }}$ uma vez que, à medida que o adolescente atravessa o processo de maturação, há uma melhora na capacidade anaeróbica envolvendo a interação de fatores morfológico, fisiológico e bioquímico (PRADO; DIAS; TROMBETTA, 2006).

Semelhante aos nossos resultados, Cooper et al. (1984) mostraram que as meninas de 12 a 17 anos apresentaram valores mais baixos para $\mathrm{VO}_{2 \operatorname{LAV}}(19 \pm 3$ $\left.\mathrm{ml} \cdot \mathrm{kg}^{-1} \cdot \mathrm{min}^{-1}\right)$ do que as meninas de 6 a II anos $\left(23 \pm 4 \mathrm{ml} \cdot \mathrm{kg}^{-1} \cdot \mathrm{min}^{-1}\right)$ e quando comparadas aos meninos $\left(27 \pm 6 \mathrm{ml} \cdot \mathrm{kg}^{-1} \cdot \mathrm{min}^{-1}\right)$ da mesma idade, sugerindo que as meninas apresentavam níveis mais elevados de lactato sanguíneo durante o exercício, além de pressões sociais e culturais que supostamente inibia a participação das adolescentes em atividades vigorosas, resultando em níveis de aptidão mais baixos, refletindo assim em menor desempenho aeróbico ao realizarem testes de esforço.

No estudo de Reybrouck et al. (1985) encontrou-se um comportamento diferente nas variáveis ao comparar com os nossos resultados. Os autores identificaram que o gênero e a idade influenciavam significativamente no $\mathrm{VO}_{2}$ máx. $\left(\mathrm{ml} . \mathrm{kg}^{-1}\right.$.

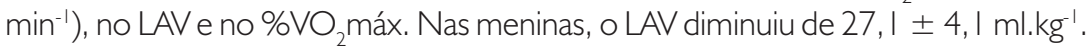
$\mathrm{min}^{-1}$ aos 9-10 anos para 24,3 $\pm 3,0 \mathrm{ml} . \mathrm{kg}^{-1} \cdot \mathrm{min}^{-1}$ aos 13-1 4 anos; nos meninos, o LAV reduziu de $34,8 \pm 6,6 \mathrm{ml} . \mathrm{kg}^{-1} \cdot \mathrm{min}^{-1}$ aos $9-10$ anos para $29, \mathrm{I} \pm 4,4 \mathrm{ml} . \mathrm{kg}^{-1}$. $\mathrm{min}^{-1}$ aos $13-14$ anos, concluindo assim que existiu uma diminuiçã̃o significativa do LAV ou do \% $\mathrm{VO}_{2}$ máx. com o aumento da idade, para ambos os gêneros, sugerindo que o aumento na capacidade anaeróbica lática aumenta com o avanço da idade. 
Neste estudo não foram encontradas diferenças estatísticas para \% $\mathrm{VO}_{2}$ máx. entre as faixas etárias em cada gênero, como também não foram encontradas diferenças entre os gêneros para cada faixa etária. Logo, esse comportamento se contrapõe ao que foi mostrado no estudo de Reybrouck et al. (1985), onde é visto uma diminuição dessa variável durante o crescimento, tanto para meninas (de 69,2 \pm 6,2 \%VO ${ }_{2}$ máx. para 53,8 \pm 6,3 \% $\mathrm{VO}_{2}$ máx.), quanto para os meninos (de 74,4 $\pm 6 \% \mathrm{VO}_{2}$ máx. para $50,5 \pm 7,5 \% \mathrm{VO}_{2}$ máx.).

Embora seja importante conhecer a $\mathrm{FC}_{\mathrm{LAV}}$ em adolescentes para o controle da intensidade do exercício, com finalidade de melhorar a aptidão para saúde ou para o rendimento esportivo (PEREZ; CARLETTI, 20 I 2), a literatura nacional apresenta poucos trabalhos (BRUNETTO et al., 2005; STELLA et al., 2005) que analisaram essa variável ao nível submáximo do teste progressivo.

Em nossos resultados observamos que a $\mathrm{FC}_{\mathrm{LAV}}$ das meninas de 10 anos apresentavam maior média ( $48 \pm 20$ bpm) quando comparadas às de outras faixas etárias. Na investigação de Rowland e Green ( 1989), a FC LAV de meninas com idade de II,5 anos foi de 17I bpm ( \pm |2), média muito acima daquela encontrada para as jovens deste estudo de faixa etária correspondente (I43 \pm 7 bpm).

Por outro lado, os meninos não apresentaram diferenças para $\mathrm{FC}_{\mathrm{LAV}}$ entre as faixas etárias. Em contrapartida, Al-Hazzaa (200 I) estudou o desenvolvimento da função cardiorrespiratória de meninos entre 7 e 15 anos e observou que a FC foi significativamente maior nos meninos de 13 a 15 anos quando comparados aos outros grupos etários. Por outro lado, Bar-Or (1983) relata que a FC submáxima nas crianças declina com a idade, sendo parcialmente explicado pela maior intensidade relativa do exercício realizado pelas crianças mais novas.

Outra variável analisada foi o percentual da FCmáx. no LAV (\%FCmáx.). Neste estudo não encontramos diferenças entre as faixas etárias para ambos os gêneros, não havendo também diferenças entre os gêneros. Contudo, não foi encontrado na literatura estudos com adolescentes que abordassem essa variável, o que torna difícil a comparação com os nossos resultados. Em adultos, o \%FCmáx. é um parâmetro de controle importante do treinamento físico, pois representa um comparativo de nível de aptidão aeróbica, importante para a prescrição de exercício (LONDEREE, 1997; EDWARDS; CLARK; MACFADYEN, 2003). A identificação do \%FCmáx. é fundamental para se trabalhar em uma intensidade ótima de esforço a fim de melhorar o estímulo cardiorrespiratório, melhorando assim o $\mathrm{VO}_{2}$ máx.

Ao analisar as variáveis da carga de trabalho durante o teste cardiopulmonar (Vel ${ }_{\text {LAV }}$ Incl ${ }_{\text {LAV }}$ ), poucos estudos apresentam os valores atingidos no LAV, enfatizando apenas a fase máxima do teste, além dos diferentes protocolos e ergômetros que dificultam a comparação dos resultados (TOURINHO FILHO et al., 1998; 
GUERRERO; NARANJO; CARRANZA, 2008; MILANO; LEITE, 2009; GIARDINI et al., 201 I).

Nessa pesquisa utilizamos o protocolo de rampa com aumento contínuo da carga de 0,5 km/h e mostramos que os meninos de 14 anos apresentaram maiores valores para a Vel LAV $(7,9 \pm 1,1 \mathrm{~km} / \mathrm{h})$ quando comparados tanto aos meninos de outras faixas etárias, quanto às meninas de mesma idade (6,8 \pm 0,9km/h). Para Incl LAV não houve diferenças entre as faixas etárias, para ambos os gêneros e, quando comparados os gêneros, apenas aos 14 anos os meninos apresentaram maiores valores que as meninas $(0,8 \pm 1,6 \%$ vs $0,3 \pm 1,3 \%)$. Nesse sentido, sugere-se que o processo de crescimento pode influenciar na eficiência do movimento, sendo significativo a partir dos 14 anos.

De acordo com a literatura (GUERRERO; NARANJO; CARRANZA, 2008; MILANO; LEITE, 2009), é possível observar que entre os gêneros, de maneira geral, os meninos apresentam médias maiores para a carga de trabalho, existindo também diferenças entre as faixas etárias.

\section{CONCLUSÕES}

presente estudo mostrou que, com base na amostra estudada, houve perda da aptidão aeróbica em nível submáximo dos adolescentes a partir dos 12 anos, apresentando uma tendência à redução do $\mathrm{VO}_{2 \operatorname{LAV}}\left(\mathrm{ml} \cdot \mathrm{kg}^{-1} \cdot \mathrm{min}^{-1}\right)$ nas meninas e uma estabilização nos meninos. Foi possível observar também uma semelhança entre meninos e meninas até os 13 anos. Após essa idade, os meninos apresentaram valores mais elevados, sugerindo uma melhora nessa aptidão.

Nossos resultados também sugerem que a aptidão aeróbica submáxima avaliada, a partir das respostas cardiorrespiratórias do teste de esforço progressivo dos adolescentes, é inferior quando comparada aos dados da literatura.

Vale ressaltar que os resultados podem servir de referência para intervenções futuras, porém a inferência dos mesmos deve ser feita cautelosamente para os demais adolescentes brasileiros considerando a diversidade étnica, cultural e social.

Ventilatory anaerobic threshold of brazilian adolescents in both sexes

ABSTRACT: In order to compare the cardiorespiratory responses in ventilatory anaerobic threshold (VAT) in relation to gender, age and published data, were evaluated 289 adolescents of both sexes, between 10 and 14 years, who underwent a cardiopulmonary test using a ramp protocol on treadmill. The VAT was identified by the methods of the V-slope and/or ventilatory equivalent of oxygen. The results showed that oxygen consumption $\left(\mathrm{VO}_{2 \mathrm{VAT}}\right)$ absolute 
was higher in boys at 13 and 14 years. The $\mathrm{VO}_{2 \text { vat }}$ relative to body weight was different only between genders aged 14 years old, and being higher in boys than girls. We conclude that there is loss of aerobic fitness in adolescents of both sexes from the age of 12 . The $\mathrm{VO}_{2}$ VAT was lower when compared with the literature.

KEYWORDS: Oxygen consumption; adolescent; physical fitness; anaerobic threshold.

\section{Umbral anaeróbico ventilatorio en adolescentes brasileños de ambos sexos}

RESUMEN: A fin de comparar las respuestas cardiorrespiratorias en el umbral anaeróbico ventilatorio (UAV) en relación con el género, la edad y los datos publicados, se evaluaron 289 adolescentes de ambos sexos, entre 10 y 14 años. Se realizó una prueba de esfuerzo cardiopulmonar utilizando un protocolo de rampa en la cinta. El UAV fue identificado por los métodos de la $\mathrm{V}$-slope y/o equivalentes ventilatorio de oxígeno. Los resultados mostraron que el consumo de oxígeno $\left(\mathrm{VO}_{2 \text { UAv }}\right.$ ) absoluta fue mayor en los varones de 13 y 14 años, la VO 2 Uav relativo al peso corporal fue diferente entre los sexos sólo 14 años, siendo mayor en los varones. En conclusión de que hay una pérdida de la aptitud aeróbica de los adolescentes de ambos sexos de 12 años de edad, con valores más bajos en comparación con la literatura. PALABRAS CLAVE: Consumo de oxígeno; adolescente; acondicionamiento físico; umbral anaeróbico.

\section{REFERÊNCIAS}

AL-HAZZAA, H. M. Development of maximal cardiorespiratory function in Saudi boys. Saudi Medical Journal, Riyadh, v. 22, n. 10, p. 875-881, oct. 2001.

ARMSTRONG, N.; TOMKINSON, G. R.; EKELUND, U. Aerobic fitness and its relationship to sport, exercise training and habitual physical activity during youth. British Journal of Sports Medicine, Loughborough, v. 45, p. 849-858, sept. 201 I.

ARMSTRONG, N.; WELSMAN, J. R. Assessment and interpretation of aerobic fitness in children and adolescents. Exercise and Sport Sciences Review, New York, v. 22, p. 435-476, jan. 1994.

ARMSTRONG, N.; WELSMAN, J. R.; KIRBY, B. J. Submaximal exercise and maturation in I2-year-olds. Journal of Sports Sciences, London, v. 17, n. 2, p. 107-14, feb. 1999.

ARMSTRONG, N.; WELSMAN, J. R. Peak oxigen uptake in relation to growth and maturation in II- to 17-year-old humans. European Journal of Applied Physiology, Berlin, v. 85, n. 6, p. 546-55I, oct. 2001 .

ASTRAND, P. O.; RODAHL, K. Textbook of work physiology. New York: McGraw-Hill, 1986.

BAR-OR, O. Pediatric sports medicine for practitioner: from physiologic principles to clinical applications. New York: Springer-Verlag, 1983. 
BEAVER, W. L.; WASSERMAN, K.; WHIPP, B. J. A new method for detecting anaerobic threshold by gas exchange. Journal of Applied Physiology, Washington, v. 60, p. 2020-2027, jun. 1986.

BRUNETTO, A. F. et al. Limiar ventilatório e variabilidade da frequência cardíaca em adolescentes. Revista Brasileira de Medicina do Esporte, São Paulo, v. I I , n. I, jan./fev. 2005.

CHINTALA, K.; EPSTEIN, M. L.; SINGH, P. Longitudinal changes in heart rate-corrected measures of exercise performance in children. Pediatric cardiology, New York, v. 29, p. 6064, jan. 2008.

COOPER, D. M. et al. Aerobic parameters of exercise as a function of body size during growth in children. Journal of Applied Physiology, Washington, v. 56, p. 628-634, mar. 1984.

CUNNINGHAM, D. A. et al. Development of cardiorespiratory function in circumpubertal boys: a longitudinal study. Journal of Applied Physiology, Washington, v. 56, n. 2, p. 302-7, feb. 1984.

DUARTE, M. F. S. Maturação física: uma revisão da literatura, com especial atenção à criança brasileira. Caderno de Saúde Pública, Rio de Janeiro, v. 9 (supl. I), p. 7I-84, 1993.

EDWARDS, A. M.; CLARK, N.; MACFADYEN, A. M. Lactate and ventilatory thresholds reflect the training status of professional soccer players where maximum aerobic power is unchanged. Journal of Sports Science \& Medicine, Bursa, v. 2, p. 23-29, mar. 2003.

GIARDINI, A. et al. Physiologic decrease of ventilatory response to exercise in the second decade of life in healthy children. American Heart Journal, St. Louis, v. I6I, n. 6, p. I214-9, jun. 2011 .

GUERRERO, L.; NARANJO, J.; CARRANZA, M. D. Influence of gender on ventilatory efficiency during exercise in Young children. Journal of Sports Sciences, London, v. 26, n. I3, p. | 455- | 457, nov. 2008.

LONDEREE, B. R. Effect of training on lactate/ventilatory thresholds: a meta-analysis. Medicine and Science in Sports and Exercise, Madison, v. 29, n. 6, p. 837-843, jun. 1997.

MANDADZHIEVA, S. et al. Anthropometric and cardiopulmonary parameters in bulgarian and romany children: cross-sectional study. Croatian Medical Journal, Zagreb, v. 46, n. 2, p. 294-301, apr. 2005.

MILANO, G. E.; LEITE, N. Comparação das variáveis cardiorrespiratórias de adolescentes obesos e não obesos em esteira e bicicleta ergométrica. Revista Brasileira de Medicina do Esporte, São Paulo, v. I 5, n. 4, p. 25 I-254, jul./ago. 2009.

MYERS, J.; BELLIN, D. Ramp exercise protocols for clinical and cardiopulmonary exercise testing. Sports Medicine, Auckland, v. 30, n. I, p. 23-29, jul. 2000. 
O'DONOVAN, G. et al. The ABC of physical activity for health: a consensus statement from the British Association of Sport and Exercise Sciences. Journal of Sports Sciences, London, $v$. 28, n. 6, p. 573-591, apr. 2010.

PEREZ, A. J.; CARLETTI, L. Identificação do limiar anaeróbico ventilatório em crianças e adolescentes: revisão da literatura. Revista Brasileira de Cineantropometria \& Desempenho Humano, Florianópolis, v. 14, n. 3, p. 343-352, maio 2012. Disponível em: <http://www. periodicos.ufsc.br/index.php/rbcdh/issue/view/l782>. Acesso em: 08 jul. 2012.

PRADO, D. M. L.; DIAS, R. G.; TROMBETTA, I. C. Comportamento das variáveis cardiovasculares, ventilatórias e metabólicas durante o exercício: diferenças entre crianças e adultos. Arquivos Brasileiros de Cardiologia, São Paulo, v. 87, p. el 49-el 55, out. 2006.

PRADO, D. M. et al. Weight loss associated with exercise training restores ventilatory efficiency in obese children. International Journal of Sports Medicine, Stuttgart, v. 30, p. 82 I-826, nov. 2009.

PRADO, D. M. et al. Exercise training associated with diet improves heart rate recovery and cardiac autonomic nervous system activity in obese children. International Journal of Sports Medicine, Stuttgart, v. 31, p. 860-865, dec. 2010.

RATEL, S. et al. Do oxidative and anaerobic energy production in exercising muscle change throughout growth and maturation? Journal of Applied Physiology, Washington, v. 109 , p. 1562-1564, nov. 2010.

REA, L. M.; PARKER, R. A. Metodologia de Pesquisa: do planejamento a execução. São Paulo: Pioneira; 2000.

REYBROUCK, T. et al. Ventilatory anaerobic threshold in healthy children. European Journal of Applied Physiology, Berlin, v. 54, p. 278-284, sept. 1985.

RODRIGUES, A. N. et al. Maximum oxygen uptake in adolescents as measured by cardiopulmonary exercise testing: a classification proposal. Jornal de Pediatria, Rio de Janeiro, v. 82, n. 6, p. 426-30, nov./dez. 2006.

RODRIGUES, A. N. et al. The association between cardiorespiratory fitness and cardiovascular risk in adolescents. Jornal de Pediatria, Rio de Janeiro, v. 83, n. 5, p. 429-435, set./out. 2007.

ROWLAND, T.M. Fisiologia do exercício na criança. São Paulo: Manole, 2008.

ROWLAND, T. W.; DELANEY, B. C.; SICONOLFI, S. F. Athlest's heart in prepubertal children. Pediatrics, Evanston, v. 79, n. 5, p. 800-804, may 1987.

ROWLAND, T. W.; GREEN, G. M. Anaerobic threshold and the determination of training target heart rates in premenarchal girls. Pediatric Cardiology, New York, v. I0, n. 2, p. 75-79, jun. 1989. 
ROWLAND, T. W.; CUNNINGHAM, L. N. Oxygen uptake plateau during maximal treadmill exercise in children. Chest, Park Ridge, v. I01, n. 2, p. 485-489, feb. 1992.

ROWLAND, T. W.; CUNNINGHAM, L. N. Development of ventilatory responses to exercise in normal white children: a longitudinal study. Chest, Park Ridge, v. I। I, n. 2, p. 327-332, feb. 1997.

SILVA, O. B.; SARAIVA, L. C. R.; SOBRAL FILHO, D. C. Teste ergométrico em crianças e adolescentes - maior tolerância ao esforço com o protocolo em rampa. Arquivos Brasileiros de Cardiologia, São Paulo, v. 89, n. 6, p. 391-397, dez. 2007.

STELLA, S. G. et al. Effects of type of physical exercise and leisure activities on the depression scores of obese Brazilian adolescent girls. Brazilian Journal of Medical and Biological Research, Ribeirão Preto, v. 38, n. II, p. 1683-1689, nov. 2005.

TOURINHO FILHO, H. et al. Velocidade de corrida no limiar anaeróbio em adolescentes masculinos. Revista Paulista de Educação Física, São Paulo, v. I2, n. I , p. 3 I -4 I , jan./jun. 1998.

WASHINGTON, R. et al. Normal aerobic and anaerobic exercise data for North American school-age children. The Journal of Pediatrics, St. Louis, v. II2, n. 2, p. 223-233, feb. 1988.

WASSERMAN, K. et al. Physiology of exercise. In: Weingberg R, editor. Principals of Exercise Testing and Interpretation. 3rd ed. Philadelphia, PA: Lippincott Williams \& Wilkins, 1999. p. 10-59.

WASSERMAN, K. et al. Prova de Esforço Princípios e Interpretação. 3. ed. Rio de Janeiro: Revinter, 2005.

WHO. WHO Expert Committee on Physical Status: the use and interpretation of anthropometry. World Health Organization technical report series. Geneva, 1995. p. 854.

WHO. Growth reference data for 5 - 19 year. WHO Reference, 2007. Disponível em: < http:// www.who.int/growthref/en/>. Acesso em: 03 jul. 2010.

ZANCONATO, S. et al. Gas exchange during exercise in obese children. European Journal of Pediatrics, Heidelberg, v. 148, n. 7, p. 614-617, jun. 1989.

Recebido em: 12 fev. 2012

Aprovado em: 14 jul. 2012

Endereço para correspondência:

Kamilla Bolonha Gomes

Rua Antiocho Carneiro de Mendonça, 170/807

Jardim Camburi

Vitória/ES

CEP 29092-130 